\title{
IRREDUCIBLE PROJECTIVE CHARACTERS OF WREATH PRODUCTS
}

\author{
XIAOLI HU AND NAIHUAN JING \\ (Communicated by Pham Huu Tiep)
}

\begin{abstract}
The irreducible character values of the spin wreath products $\widetilde{\Gamma}_{n}=$ $\Gamma \imath \widetilde{S}_{n}$ of the symmetric group and a finite group $\Gamma$ are completely determined for arbitrary $\Gamma$.
\end{abstract}

\section{INTRODUCTION}

The symmetric group has been one of the core materials in mathematics and theoretical physics since Frobenius developed representation theory of finite groups. Using the duality between $S_{n}$ and $\mathrm{GL}_{m}$, Schur found the complete set of invariants for the general linear group and revolutionized the theory of symmetric functions using the Schur functions in his dissertation. In the thirties, Specht 13 . generalized Schur and Frobenius' theory to the wreath products of the symmetric group and any finite group. More recently Macdonald reformulated Specht's theory in the classic monograph [6].

The double covering groups $\widetilde{S}_{n}$ of the symmetric group have many similar and interesting properties as shown by Schur in [11. In that seminal paper, Schur generalized Frobenius' theory and introduced the famous Schur Q-functions. Schur's character theory of $\widetilde{S}_{n}$ consists of two parts. The first part of the character values on conjugacy classes associated to partitions with odd integer parts are exactly given by an analogous Frobenius formula in terms of the Schur Q-functions; the second part of character values on strict partitions was solved with the help of twisted tensor products of Clifford algebras. In the same direction, Morris [7 formulated an iterative rule for computing the spin character values of the symmetric group, and Nazarov [9] constructed all irreducible representations of the spin group. Józefiak [5] also computed the projective character values for a related double covering group of the hyperoctahedral group using similar techniques.

In [1], the second author and collaborators determined all irreducible character values on even conjugacy classes of the spin wreath products $\widetilde{\Gamma}_{n}$ by vertex operator calculus in the context of the spin McKay correspondence. The character values generalize the first part of Schur's theory on $\widetilde{S}_{n}$. The key was to show that the character values at conjugacy classes of even colored partitions (with odd integer parts) are given by matrix coefficients of certain products of twisted vertex operators. However, the other character values on odd strict partition-valued functions

Received by the editors February 1, 2013 and, in revised form, June 3, 2013, June 4, 2013, and July 9, 2013.

2010 Mathematics Subject Classification. Primary 20C25; Secondary 20C30, 20E22.

Key words and phrases. Wreath products, spin groups, projective characters. 
could not be explained in the context of the McKay correspondence. To the authors' knowledge, other available methods such as the Hopf algebraic approach [2] seem not helpful either.

The knowledge of the remaining character values for $\widetilde{\Gamma}_{n}$ would be an analog of the second part in Schur's pioneering work [11. In the case of $\Gamma$ being abelian, we solved the problem in [3] by using the Mackey-Wigner method of little groups (cf. [12]) to construct all irreducible spin representations of the wreath products (see also [8] for some cases). However, the method of little groups does not work in the most general case for arbitrary finite group $\Gamma$. It seems that a new method is needed for determination of the irreducible characters.

The purpose of this paper is to complete the character theory of the spin wreath products $\widetilde{\Gamma}_{n}$ and compute the missing part of the character table of $\widetilde{\Gamma}_{n}$ for any finite group $\Gamma$. We will construct all irreducible characters and, in particular, provide explicit formulas for character values on the conjugacy classes of the second type. It turns out that spin character values in this part can be nonzero for more than two conjugacy classes in contrast to Schur's case, nevertheless they are still sparsely zero. We note that the exhaustion method is used to pick up all nonzero character values, which bears some similarity to Schur's original method.

\section{Projective Representations of $\widetilde{\Gamma}_{n}$}

2.1. Spin wreath products. According to Schur, there are two nonisomorphic double covering groups of the symmetric group $S_{n}$ when $n \geq 4$ and $n \neq 6$. But their representations are in complete one-to-one correspondence. We fix one of them, and let the spin symmetric group $\widetilde{S}_{n}$ be the finite group generated by $z$ and $t_{i}(i=1, \cdots, n-1)$ with the relations:

$$
z^{2}=1, t_{i}^{2}=\left(t_{i} t_{i+1}\right)^{3}=z, z t_{i}=t_{i} z, t_{i} t_{j}=z t_{j} t_{i} \quad(|i-j|>1) .
$$

Let $\theta_{n}$ be the homomorphism from $\widetilde{S}_{n}$ to $S_{n}$ sending $t_{i}$ to the transposition $(i, i+1)$ and $z$ to 1 . This says that $\widetilde{S}_{n}$ is a central extension of $S_{n}$ by the cyclic group $\mathbb{Z}_{2}$.

The spin group $\widetilde{S}_{n}$ has a cycle presentation à la Conway (see [14]). For $i<j$, the transposition $[i j]$ is defined as $[i j]=t_{j-1} \cdots t_{i+1} t_{i} t_{i+1} \cdots t_{j-1}$. Here if $j=i+1$, we take $t_{i}=[i, i+1]$. Then for $i_{1}<i_{2}<\cdots<i_{k} \leq n$, we define $\left[i_{1} i_{2} \cdots i_{k}\right]=$ $\left[i_{1} i_{2}\right]\left[i_{2} i_{3}\right] \cdots\left[i_{k-1} i_{k}\right]$. Finally for a permutation $\sigma \in S_{k}$ we define

$$
\left[i_{\sigma(1)} i_{\sigma(2)} \cdots i_{\sigma(k)}\right]=z^{l(\sigma)}\left[i_{1} i_{2} \cdots i_{k}\right] .
$$

Given a permutation $w \in S_{n}$, we can fix its cycle product as follows. First, each cycle is written as a word lexicographically by rotating its content, then we rearrange the order of the cycles lexicographically to obtain a unique presentation $w=\prod_{i=1}^{l}\left(a_{i 1} \cdots a_{i \lambda_{i}}\right)$, where the $\lambda_{i}$ are the lengths of the cycles. We then define the element $t_{w}=\prod_{i=1}^{l}\left[a_{i 1} \cdots a_{i \lambda_{i}}\right] \in \widetilde{S}_{n}$. Note that $\theta_{n}^{-1}(w)=z^{p} t_{w}$. One also has that $t_{w_{1}} t_{w} t_{w_{1}}^{-1}=z^{p} t_{w_{1} w w_{1}^{-1}}$ for $w, w_{1} \in S_{n}$, where $p=0$ or 1 . Similarly for a partition $\rho$ we denote $t_{\rho}=t_{w(\rho)}$, where $w(\rho)=\left(1 \cdots \rho_{1}\right) \cdots\left(n-\rho_{l}+1 \cdots n\right) \in S_{n}$.

For a positive integer $n$ and a finite group $\Gamma$, let $\Gamma^{n}=\Gamma \times \cdots \times \Gamma$ be the $n$-fold direct product of $\Gamma$, and let $\Gamma^{0}=1$. The spin group $\widetilde{S}_{n}$ acts on $\Gamma^{n}$ by permuting the components:

$$
t_{w}\left(g_{1}, \cdots, g_{n}\right)=\left(g_{w^{-1}(1)}, \cdots, g_{w^{-1}(n)}\right), z\left(g_{1}, \cdots, g_{n}\right)=\left(g_{1}, \cdots, g_{n}\right) .
$$


The spin wreath product $\widetilde{\Gamma}_{n}=\Gamma 2 \widetilde{S}_{n}$ is the semidirect product

$$
\widetilde{\Gamma}_{n}=\Gamma^{n} \rtimes \widetilde{S}_{n}=\left\{(g, t) \mid g=\left(g_{1}, \cdots, g_{n}\right) \in \Gamma^{n}, t \in \widetilde{S}_{n}\right\}
$$

with the multiplication $(g, t) \cdot(h, s)=(g t(h), t s)$. The quotient group $\widetilde{\Gamma}_{n} /\langle z\rangle$ is isomorphic to the semidirect product $\Gamma_{n}=\Gamma^{n} \rtimes S_{n}$, and the canonical homomorphism $\theta_{n}$ from $\widetilde{\Gamma}_{n}$ to $\Gamma_{n}$ sends $\left(g, t_{i}\right) \mapsto(g,(i, i+1))$ and $(g, z) \mapsto(g, 1)$. For simplicity, we have used the same symbol for the homomorphism $\theta_{n}$ for the wreath product.

We can define a parity $p$ for $\widetilde{\Gamma}_{n}$ by (here $g \in \Gamma^{n}, t_{i} \in \widetilde{S}_{n}$ )

$$
p\left(g, t_{i}\right)=1(1 \leq i \leq n-1), \quad p(g, z)=0 .
$$

This agrees with the usual parity for $\widetilde{S}_{n}$ when $\Gamma$ is the trivial group. Therefore, the group algebra $\mathbb{C}\left[\widetilde{\Gamma}_{n}\right]$ has a superalgebra structure, and $\left.\mathbb{C}[\Gamma\} \widetilde{A}_{n}\right]$ is the even subspace.

2.2. Conjugacy classes. Let $\Gamma_{*}=\left\{c^{i} \mid i=0,1, \cdots, r\right\}$ be the set of conjugacy classes of $\Gamma$ and denote by $\Gamma^{*}=\left\{\gamma_{i} \mid i=0,1, \cdots, r\right\}$ the set of irreducible characters of $\Gamma$. Let $\zeta_{c}$ be the order of the centralizer of an element in the conjugacy class $c \in \Gamma_{*}$; then the order of the conjugacy class $c$ is $|\Gamma| / \zeta_{c}$. Here for a finite set $X$ we denote by $|X|$ its cardinality. In the following we follow Macdonald's notations [6].

A partition-valued function $\rho=(\rho(c))_{c \in \Gamma_{*}}$ defined on $\Gamma_{*}$ consists of $\left|\Gamma_{*}\right|$ partitions indexed by conjugacy classes $c \in \Gamma_{*}$. The weight of $\rho$ is defined by $\|\rho\|=$ $\sum_{c \in \Gamma_{*}}|\rho(c)|$, and the length is given by $l(\rho)=\sum_{c \in \Gamma_{*}} l(\rho(c))$. It helps to visualize $\rho$ as a colored partition in which each subpartition $\rho(c)$ is colored by $c$. Let $\mathcal{P}\left(\Gamma_{*}\right)$ be the set of partition-valued functions indexed by $\Gamma_{*}$. It is well known that the conjugacy classes of $\Gamma_{n}$ are parameterized by $\mathcal{P}\left(\Gamma_{*}\right)$. For an element $(g, \sigma) \in \Gamma_{n}$, the permutation $\sigma$ gives rise to a cycle partition $\lambda=\left(\lambda_{1}, \lambda_{2}, \cdots\right)$. For each part $\lambda_{i}=k$, which corresponds to the cycle $\left(i_{1} i_{2} \cdots i_{k}\right)$, we associate the cycle-product $g_{i_{k}} g_{i_{k-1}} \cdots g_{i_{1}} \in \Gamma$. If the cycle-product belongs to the conjugacy class $c$, then we color this part $\lambda_{i}$ by $c$ which turns $\lambda$ into a colored partition. In this way we get the parametrization of conjugacy classes of $\Gamma_{n}$ by $\mathcal{P}\left(\Gamma_{*}\right)$. For $\rho=(\rho(c))_{c \in \Gamma_{*}} \in \mathcal{P}_{n}\left(\Gamma_{*}\right)$, let $C_{\rho}$ be the corresponding conjugacy class in $\Gamma_{n}$.

We denote by $\mathcal{S P}\left(\Gamma_{*}\right)$ the set of partition-valued functions $(\rho(c))_{c \in \Gamma_{*}}$ in $\mathcal{P}\left(\Gamma_{*}\right)$ such that each partition $\rho(c)$ is strict, i.e., $\rho(c)$ has distinct parts. Let $\mathcal{O P}\left(\Gamma_{*}\right)$ be the set of partition-valued functions $(\rho(c))_{c \in \Gamma_{*}}$ on $\Gamma_{*}$ such that all parts of the partitions $\rho(c)$ are odd integers.

For each partition $\lambda$ we define the parity $d(\lambda)=|\lambda|-l(\lambda)$. Similarly, for a partition-valued function $\rho=(\rho(c))_{c \in \Gamma_{*}}$, we define $d(\rho)=\|\rho\|-l(\rho)$. Then $\rho$ is even (resp. odd) if $d(\rho)$ is even (resp. odd). We set $\mathcal{P}_{n}^{0}\left(\Gamma_{*}\right)$ (resp. $\left.\mathcal{P}_{n}^{1}\left(\Gamma_{*}\right)\right)$ to be the collections of even (resp. odd) partition-valued functions $\rho$ on $\Gamma_{*}$ such that $\|\rho\|=n$. As a convention we denote $\mathcal{S P}_{n}^{i}\left(\Gamma_{*}\right)=\mathcal{P}_{n}^{i}\left(\Gamma_{*}\right) \cap \mathcal{S P}\left(\Gamma_{*}\right)$ and $\mathcal{O P} \mathcal{P}_{n}\left(\Gamma_{*}\right)=\mathcal{P}_{n}\left(\Gamma_{*}\right) \cap \mathcal{O P}\left(\Gamma_{*}\right)$ for $i \in\{0,1\}$. When $\Gamma_{*}$ just consists of a single element, $\mathcal{P}\left(\Gamma_{*}\right)$ will be simply written as $\mathcal{P}$. Similarly, we have notations such as $\mathcal{O P}{ }_{n}, \mathcal{S P}_{n}$, and $\mathcal{S P}_{n}^{i}$.

2.3. Split conjugacy classes. An element $\widetilde{x} \in \widetilde{\Gamma}_{n}$ is called nonsplit if $\widetilde{x}$ is conjugate to $z \widetilde{x}$. Otherwise $\widetilde{x}$ is said to be split. An element $x \in \Gamma_{n}$ is called split if $\theta_{n}^{-1}(x)$ is split. A conjugacy class of $\widetilde{\Gamma}_{n}$ is called split if its elements are split. It is known that the conjugacy class $C_{\rho}$ of $\Gamma_{n}$ splits if and only if the preimage $\theta_{n}^{-1}\left(C_{\rho}\right)=: D_{\rho}$ splits into two conjugacy classes in $\widetilde{\Gamma}_{n}$. 
For a partition $\lambda=\left(1^{m_{1}} 2^{m_{2}} 3^{m_{3}} \ldots\right)$ of $n$, we denote by $z_{\lambda}=\prod_{i>1} i^{m_{i}} m_{i}$ ! the order of the centralizer of the permutation with cycle type $\lambda$ in $S_{n}$. For each partition-valued function $\rho=(\rho(c))_{c \in \Gamma_{*}}$, we define

$$
Z_{\rho}=\prod_{c \in \Gamma_{*}} z_{\rho(c)} \zeta_{c}^{l(\rho(c))},
$$

which is the order of the centralizer of an element of conjugacy type $\rho=(\rho(c))_{c \in \Gamma_{*}}$ in $\Gamma_{n}$. The order of the centralizer of an element of conjugacy type $\rho$ in $\widetilde{\Gamma}_{n}$ is given by

$$
\widetilde{Z}_{\rho}=\left\{\begin{aligned}
2 Z_{\rho}, & C_{\rho} \text { is split } \\
Z_{\rho}, & C_{\rho} \text { is nonsplit. }
\end{aligned}\right.
$$

For each split conjugacy class $C_{\rho}$ in $\Gamma_{n}$, we define the conjugacy class $D_{\rho}^{+}$in $\widetilde{\Gamma}_{n}$ to be the conjugacy class containing the element $\left(g, t_{\rho}\right)$ and define $D_{\rho}^{-}=z D_{\rho}^{+}$; then $D_{\rho}=D_{\rho}^{+} \cup D_{\rho}^{-}$.

As usual, a representation $\pi$ of $\widetilde{\Gamma}_{n}$ is called $\operatorname{spin}$ if $\pi(z)=-i d$; then its character is a projective character of $\Gamma_{n}$. It is clear that the character of a spin representation of $\widetilde{\Gamma}_{n}$ is determined by its values on split conjugacy classes. By a standard result [1,10 the conjugacy class of $\widetilde{\Gamma}_{n}$ is split in $\widetilde{\Gamma}_{n}$ if and only if either $\rho \in \mathcal{O} \mathcal{P}_{n}\left(\Gamma_{*}\right)$ or $\rho \in \mathcal{S P}_{n}^{1}\left(\Gamma_{*}\right)$. In particular, the split conjugacy classes of $\widetilde{S}_{n}$ (i.e., when $\Gamma$ is the trivial group) are parameterized either by partitions with odd integers or by odd strict partitions. By Euler's theorem the number of strict partitions is equal to the number of odd partitions, therefore the total number of such conjugacy classes of $\widetilde{\Gamma}_{n}$ is given by $\left|\mathcal{S P}_{n}^{0}\left(\Gamma_{*}\right)\right|+2\left|\mathcal{S} \mathcal{P}_{n}^{1}\left(\Gamma_{*}\right)\right|$.

\section{The IRREDUCiBle SPIN CHARACTER TABle OF $\widetilde{\Gamma}_{n}$}

3.1. Schur's theory of $\widetilde{S}_{n}$. Like the symmetric group $S_{n}$, nontrivial projective (spin) characters of $\widetilde{S}_{n}$ are parameterized by strict partitions $\lambda$ of $n$. One can classify spin characters into the so-called double spin and associate spin characters. The associated character $\chi^{\prime}$ of a spin character is defined to be $\chi^{\prime}=\operatorname{sgn} \cdot \chi$, where $s g n$ is the sign character. If $\chi^{\prime}=\chi$, then we say $\chi$ is a double spin character (or self-associated). For $\nu \in \mathcal{S P}_{n}$ and $d(\nu)=n-l(\nu)$ even, there corresponds a unique irreducible (double) spin character $\Delta_{\nu}$; for $d(\nu)$ odd, there corresponds a pair of irreducible (associate) spin characters $\Delta_{\nu}^{ \pm}$for $\widetilde{S}_{n}$. Schur [1] showed that there was an analogous Frobenius formula for the spin character values at the even conjugacy classes indexed by partitions with odd integers. For $\nu \in \mathcal{S P}$ the Schur $Q$-function $Q_{\nu}$ is defined by

$$
Q_{\nu}\left(x_{1}, \cdots, x_{n}\right)=2^{l} \sum_{w \in S_{n} / S_{n-l}} x_{w(1)}^{\nu_{1}} \cdots x_{w(n)}^{\nu_{n}} \prod_{i<j} \frac{x_{w(i)}+x_{w(j)}}{x_{w(i)}-x_{w(j)}},
$$

where $n \geq l=l(\nu)$ and $S_{n-l}$ acts on $x_{l+1}, \cdots, x_{n}$. It is known that $Q_{\nu}$ is a polynomial in power sum symmetric functions $p_{1}, p_{3}, p_{5}, \cdots$. As usual we will denote $p_{\alpha}=p_{\alpha_{1}} p_{\alpha_{2}} \cdots$ for a partition $\alpha$.

Schur showed that nontrivial values of the spin character $\Delta_{\nu}$ at even conjugacy classes are given by

$$
Q_{\nu}=\sum_{\alpha \in \mathcal{O P}_{n}} 2^{\left[\frac{l(\nu)+l(\alpha)+\bar{d}(\nu)}{2}\right]} z_{\alpha}^{-1} \Delta_{\nu}(\alpha) p_{\alpha},
$$


where $[a]$ denotes the largest integer $\leq a$ and $\bar{d}(\nu)$ is equal to 0 (resp. 1) if $d(\nu)$ is even (resp. odd). Schur further proved the following results.

Theorem 3.1 ([1]). (i) For $n-l(\nu)$ even, the character $\Delta_{\nu}$ of $\widetilde{S}_{n}$ is determined by $\left\{\Delta_{\nu}(\alpha) \mid \alpha \in \mathcal{O P}_{n}\right\}$ (given by (3.1)) and $\Delta_{\nu}(\mu)=0$ for $\mu \notin \mathcal{O P} \mathcal{P}_{n}$.

(ii) For $n-l(\nu)$ odd, the character $\Delta_{\nu}$ of $\widetilde{S}_{n}$ is determined by $\left\{\Delta_{\nu}(\alpha) \mid \alpha \in \mathcal{O} \mathcal{P}_{n}\right\}$ (given by (3.1)) and $\Delta_{\nu}(\nu)=(\sqrt{-1})^{(n-l(\nu)+1) / 2} \sqrt{\nu_{1} \cdots \nu_{k} / 2}$ for $\nu=\left(\nu_{1}, \cdots, \nu_{k}\right)$; $\Delta_{\nu}(\mu)=0$ for $\mu \neq \nu$ and $\mu \in \mathcal{S P}_{n}^{1}$. Moreover, $\left(\Delta_{\nu}\right)^{\prime}(\alpha)=\Delta_{\nu}(\alpha)$ for $n-l(\alpha)$ even and $\left(\Delta_{\nu}\right)^{\prime}(\alpha)=-\Delta_{\nu}(\alpha)$ for $n-l(\alpha)$ odd.

For $\widetilde{\Gamma}_{n}$, the values of nontrivial spin characters at the conjugacy classes associated to $\rho$ for $\rho \in \mathcal{O P}_{n}\left(\Gamma_{*}\right)$ are given by Frenkel-Jing-Wang [1].

Lemma 3.2. For an irreducible spin $\widetilde{\Gamma}_{n}$-character $\chi_{\lambda}$ with type $\lambda \in \mathcal{S P}_{n}^{1}\left(\Gamma^{*}\right)$ and a subset $G$ of $\left(\widetilde{\Gamma}_{n}\right)_{*}$, set $\left\langle\chi_{\gamma}, \chi_{\gamma}\right\rangle_{G}=\sum_{D_{\rho} \in G} \frac{1}{\left|\widetilde{Z}_{\rho}\right|}\left|\chi\left(D_{\rho}\right)\right|^{2}$. Then $\left\langle\chi_{\gamma}, \chi_{\gamma}\right\rangle_{\mathcal{O} \mathcal{P}_{n}\left(\Gamma_{*}\right)}=$ $\left\langle\chi_{\gamma}, \chi_{\gamma}\right\rangle_{\mathcal{S P}}^{1}\left(\Gamma_{*}\right)=\frac{1}{2}$ (see [1]).

Proof. Since $\chi_{\lambda}^{\prime}(x)=(-1)^{\operatorname{deg}(x)} \chi_{\lambda}(x)$ for $x \in \widetilde{\Gamma}_{n}$, we have that

$$
\begin{aligned}
2 & =\left\langle\chi_{\lambda}+\chi_{\lambda}^{\prime}, \chi_{\lambda}+\chi_{\lambda}^{\prime}\right\rangle_{\widetilde{\Gamma}_{n}} \\
& =\left(\sum_{\rho \in \mathcal{O} \mathcal{P}_{n}\left(\Gamma_{*}\right)}+\sum_{\rho \in \mathcal{S} \mathcal{P}_{n}^{1}\left(\Gamma_{*}\right)}\right) \frac{1}{\widetilde{Z}_{\rho}}\left|\left(\chi_{\lambda}+\chi_{\lambda}^{\prime}\right)\left(D_{\rho}\right)\right|^{2} \\
& =\sum_{\rho \in \mathcal{O P}_{n}\left(\Gamma_{*}\right)} \frac{1}{\widetilde{Z}_{\rho}} \cdot 4\left|\chi_{\lambda}\left(D_{\rho}\right)\right|^{2} .
\end{aligned}
$$

Therefore, $\sum_{\rho \in \mathcal{O} \mathcal{P}_{n}\left(\Gamma_{*}\right)} \frac{1}{\widetilde{Z}_{\rho}}\left|\chi_{\lambda}\left(D_{\rho}\right)\right|^{2}=\sum_{\rho \in \mathcal{S} \mathcal{P}_{n}^{1}\left(\Gamma_{*}\right)} \frac{1}{\widetilde{Z}_{\rho}}\left|\chi_{\lambda}\left(D_{\rho}\right)\right|^{2}=\frac{1}{2}$.

Table 1 shows the status of character values. Part $D$ indicates what we will compute in this paper: $\chi_{\gamma}\left(D_{\rho}^{ \pm}\right)$for $\rho \in \mathcal{S P}_{n}^{1}\left(\Gamma_{*}\right)$.

TABLE 1. The spin character table for $\widetilde{\Gamma}_{n}$

\begin{tabular}{ccc}
\hline Character $\backslash$ Class : & $\rho \in \mathcal{O P}{ }_{n}\left(\Gamma_{*}\right)$ & $\rho \in \mathcal{S P}_{n}^{1}\left(\Gamma_{*}\right)$ \\
\hline$\lambda \in \mathcal{S P}_{n}^{0}\left(\Gamma^{*}\right), \chi_{\lambda}:$ & A: known & C: 0 \\
$\left\langle\chi_{\lambda}, \chi_{\lambda}\right\rangle:$ & 1 & 0 \\
\hline$\lambda \in \mathcal{S P} \mathcal{P}_{n}^{1}\left(\Gamma^{*}\right), \chi_{\lambda}:$ & B: known & D: this paper \\
$\left\langle\chi_{\lambda}, \chi_{\lambda}\right\rangle:$ & $1 / 2$ & $1 / 2$ \\
\hline
\end{tabular}

3.2. Decomposition of colored partitions. When $\Gamma$ is a finite abelian group, we used the Mackey-Wigner method of little groups to decompose the action of $S_{n}$ on the characters of $\Gamma^{n}$. It turns out that the invariant subgroup of each $S_{n}$-orbit is a Young subgroup of $S_{n}$ and vice versa. Then we can construct all spin irreducible representations indexed by strict partition-valued functions by induction, and show that the character values are sparsely zero and the nonzero values are given according to how the partitions are supported on various conjugacy classes (see [3]). This method is no longer available when $\Gamma$ is an arbitrary finite group. Next we use a different method to compute spin character values on odd strict partition-valued functions for a general finite group $\Gamma$. 
We discuss the conjugacy classes generated by Young subgroups. For $\nu=$ $\left(\nu_{\gamma}\right)_{\gamma \in \Gamma^{*}} \in \mathcal{S P}\left(\Gamma^{*}\right)$, we first erase the coloring of $\nu_{\gamma_{i}}$ and reassign colors arbitrarily from $\Gamma_{*}$. Suppose $\nu_{\gamma_{i}}=\left(\nu_{1}^{i}, \nu_{2}^{i}, \cdots, \nu_{s}^{i}\right)$ as an ordinary strict partition. Then for any composition $c(\underline{i})=\left(c^{i_{1}}, \cdots, c^{i_{s}}\right)$ from $\Gamma_{*}=\left\{c^{0}, \cdots, c^{r}\right\}$, we assign the colors $c^{i_{1}}, \cdots, c^{i_{s}}$ consecutively to the parts of $\nu_{\gamma_{i}}$ to get a colored partition $\nu^{i}(\underline{c})$ of the shape $\nu^{i}$. The resulted multi-colored partition will be denoted by $\nu^{I}$, where $I \in\{0, \cdots, r\}^{l(\nu)}$. We let $[\nu]$ be the collection of all these multi-colored partition-valued functions, which are parameterized by mappings $I:\{1,2, \cdots, l(\nu)\} \rightarrow\{0, \cdots, r\}$. Therefore the cardinality of $[\nu]$ is $(r+1)^{l(\nu)}$. See the following example.
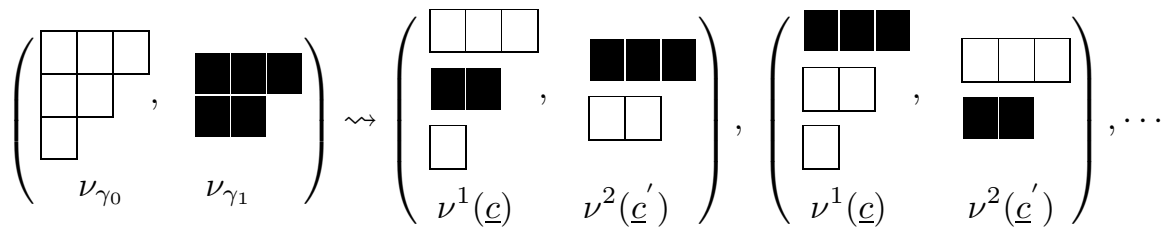

3.3. Spin supermodules vs. spin modules. A spin $\widetilde{\Gamma}_{n}$-module $V$ becomes a spin supermodule when $c h_{V}(x)=0$ for all odd elements $x$. According to [5] there are two basic types of simple supermodules: type $M$ or $Q$, corresponding to our double spin and a pair of associated spin modules when forgetting the $\mathbb{Z}_{2}$-gradation. Moreover, all double spin and associate spin modules are realized in this way.

For a strict partition-valued function $\lambda=\left(\lambda_{\gamma}\right)_{\gamma \in \Gamma^{*}} \in \mathcal{S P}_{n}\left(\Gamma^{*}\right)$, let $J_{\lambda}=\{\gamma \in$ $\Gamma^{*} \mid \lambda_{\gamma}$ is odd strict $\}$ and $J_{\lambda}^{\prime}=\Gamma^{*}-J_{\lambda}=\left\{\gamma \in \Gamma^{*} \mid \lambda_{\gamma}\right.$ is even strict $\}$. Let $U_{\gamma}$ be the irreducible $\Gamma$-module associated with the irreducible character $\gamma \in \Gamma^{*}$. Suppose $V_{\lambda_{\gamma}}$ is the irreducible spin $\widetilde{S}_{\left|\lambda_{\gamma}\right|}$-supermodule determined by the strict partition $\lambda_{\gamma}$; then $U_{\gamma}^{\otimes\left|\lambda_{\gamma}\right|} \otimes V_{\lambda_{\gamma}}$ is a spin $\widetilde{\Gamma}_{\left|\lambda_{\gamma}\right|}$-supermodule. Let $\widetilde{\Gamma}_{\lambda}$ be the nontrivial double cover of the Young subgroup $\Gamma^{n} \rtimes S_{\left(\left|\lambda_{\gamma_{0}}\right|, \cdots,\left|\lambda_{\gamma_{r}}\right|\right)}$. Then by [1], the supertensor product

$$
\hat{\bigotimes}_{\gamma \in \Gamma^{*}}\left(U_{\gamma}^{\otimes\left|\lambda_{\gamma}\right|} \otimes V_{\lambda_{\gamma}}\right)
$$

decomposes completely into $2^{\left[\frac{\left|J_{\lambda}\right|}{2}\right]}$ copies of an irreducible spin $\widetilde{\Gamma}_{\lambda}$-supermodule. Denote this irreducible supermodule by $W_{\lambda}$.

Proposition 3.3. The underlying $\widetilde{\Gamma}_{n}$-module of the induced supermodule $\operatorname{Ind}_{\widetilde{\Gamma}_{\lambda}} \widetilde{\Gamma}_{n} W_{\lambda}$ is an irreducible double spin $\widetilde{\Gamma}_{n}$-module or a direct sum of two associated irreducible $\widetilde{\Gamma}_{n}$-modules according to whether the supermodule is of type $M$ or $Q$ respectively. In terms of partitions this corresponds to whether $d(\lambda)=n-l(\lambda)$ is even or odd.

Proof. This is true in a more general context. Each irreducible $\widetilde{\Gamma}_{n}$-supermodule of type $M$ (resp. $Q$ ) is an irreducible double spin (resp. a pair of associated spin) $\widetilde{\Gamma}_{n}$-module(s). Suppose that the underlying $\widetilde{\Gamma}_{n}$-module of our irreducible $\widetilde{\Gamma}_{n}$-supermodule $V=\operatorname{Ind} \widetilde{\Gamma}_{\lambda} \widetilde{\Gamma}_{n} W_{\lambda}$ decomposes into a direct sum of irreducible $\widetilde{\Gamma}_{n^{-}}$ modules:

$$
V=\sum_{i=1}^{m} V_{i} \oplus \sum_{j=1}^{q}\left(W_{j} \oplus W_{j}^{\prime}\right),
$$

where the $V_{i}$ are irreducible double spin modules, and $W_{j}$ and $W_{j}^{\prime}$ are irreducible associate spin modules. It follows from the general theory [4] of double spin and 
associate spin modules that, as an $\Gamma$ 々 $\widetilde{A}_{n}$-module, $\operatorname{Res}\left(V_{i}\right)$ decomposes into $V_{i}^{\prime} \oplus V_{i}^{\prime \prime}$, while $\operatorname{Res}\left(W_{j}\right)$ or $\operatorname{Res}\left(W_{j}^{\prime}\right)$ remains irreducible. Thus we will have

$$
\langle V, V\rangle_{\Gamma \imath \widetilde{A}_{n}}=2 m+2 q .
$$

On the other hand, we know that $\langle V, V\rangle_{\widetilde{\Gamma}_{n}}=1$ or 2 according to the spin supermodule $V$ being of type $M$ or $Q$ by vertex operator calculus [1]. This means that $\langle V, V\rangle_{\Gamma \imath \widetilde{A}_{n}}=2$, so we must have that either $m=1$ or $q=1$.

3.4. Construction of irreducible spin characters. Let $\tilde{\Delta}_{\lambda_{\gamma}}$ be the character of the supermodule $V_{\lambda_{\gamma}}$. Then $\hat{\otimes}_{\gamma \in \Gamma^{*}}\left(\gamma^{\otimes}\left|\lambda_{\gamma}\right| \otimes \tilde{\Delta}_{\lambda_{\gamma}}\right)$ is the character of the supertensor product $\hat{\bigotimes}_{\gamma \in \Gamma^{*}}\left(U_{\gamma}^{\otimes\left|\lambda_{\gamma}\right|} \otimes V_{\lambda_{\gamma}}\right)$. In order to consider the ordinary spin characters of $\widetilde{\Gamma}_{\lambda}$, Schur defined the starred tensor product $\otimes_{\gamma \in \Gamma^{*}}\left(\gamma^{\otimes}\left|\lambda_{\gamma}\right| \otimes \Delta_{\lambda_{\gamma}}\right)$ to be an underlying ordinary irreducible component of $\hat{\bigotimes}_{\gamma \in \Gamma^{*}}\left(\gamma^{\otimes}\left|\lambda_{\gamma}\right| \otimes \tilde{\Delta}_{\lambda_{\gamma}}\right)$ (see [11]). Let $\tilde{\chi}_{\lambda}$ (resp. $\tilde{\Delta}_{\lambda_{\gamma}}$ ) be the character of irreducible supermodule $W_{\lambda}$ (resp. $V_{\lambda_{\gamma}}$ ). It is known that $\tilde{\chi}_{\lambda}$ (resp. $\tilde{\Delta}_{\lambda_{\gamma}}$ ) is also an irreducible ordinary character when $d(\lambda)$ (resp. $\left.d\left(\lambda_{\gamma}\right)\right)$ is even. We denote it by $\chi_{\lambda}$ (resp. $\Delta_{\lambda_{\gamma}}$ ) when it is regarded as an ordinary character, while $\tilde{\chi}_{\lambda}$ (resp. $\tilde{\Delta}_{\lambda_{\gamma}}$ ) decomposes into two ordinary irreducible characters $\chi_{\lambda}$ and $\chi_{\lambda}^{\prime}$ (resp. $\Delta_{\lambda_{\gamma}}$ and $\left.\Delta_{\lambda_{\gamma}}^{\prime}\right)$ when $d(\lambda)\left(\right.$ resp. $\left.d\left(\lambda_{\gamma}\right)\right)$ is odd.

Remark 3.4. Let $\rho=\rho^{0} \cup \cdots \cup \rho^{r}$ be a union of partition-valued functions on $\Gamma_{*}$ whose parts are a union of those of partition-valued functions $\rho^{0}, \cdots, \rho^{r}$ on $\Gamma_{*}$. Then $D_{\rho}$ splits into two conjugacy classes of $\widetilde{\Gamma}_{\left(\left|\rho^{0}\right|, \cdots,\left|\rho^{r}\right|\right)}$ if and only if

(1) $\rho=\rho^{0} \cup \cdots \cup \rho^{r} \in \mathcal{O} \mathcal{P}_{\left|\rho^{0}\right|+\cdots+\left|\rho^{r}\right|}\left(\Gamma_{*}\right)$ or

(2) $\rho^{i} \in \mathcal{S P}_{\left|\rho^{i}\right|}\left(\Gamma_{*}\right)$ for $0 \leq i \leq r$ and $\|\rho\|-l(\rho)$ is odd.

For brevity, we have used the numbers $0,1, \cdots, r$ by $\gamma \in \Gamma^{*}$ to index each subpartition-valued function in $\rho$. More precisely, we denote $\rho_{\gamma_{j}}:=\rho^{j}$. For $\left(g, t_{\rho}\right) \in \widetilde{\Gamma}_{\lambda}$ with $\rho=\rho^{0} \cup \cdots \cup \rho^{r}$, one has 3 ]

$$
\begin{aligned}
& \chi_{\lambda}\left(g, t_{\rho}\right)=\otimes_{\gamma \in \Gamma^{*}}\left(\gamma \otimes\left|\lambda_{\gamma}\right| \otimes \Delta_{\lambda_{\gamma}}\right)\left(g, t_{\rho}\right) \\
= & 2^{\left[\frac{\left|J_{\lambda}\right|}{2}\right]}(\sqrt{-1})^{\left[\frac{\left|J_{\lambda}\right|}{2}\right] \cdot \prod_{\gamma \in J_{\lambda}} d\left(t_{\rho_{\gamma}}\right)} \cdot \prod_{\gamma \in \Gamma^{*}} \gamma^{\otimes\left|\lambda_{\gamma}\right|} \otimes \Delta_{\lambda_{\gamma}}\left(g, t_{\rho_{\gamma}}\right) .
\end{aligned}
$$

Let $\tilde{\chi}_{\lambda} \uparrow$ be the induced character of $\tilde{\chi}_{\lambda}$ from $\widetilde{\Gamma}_{\lambda}$ to $\widetilde{\Gamma}_{n}$. We have $\tilde{\chi}_{\lambda} \uparrow=\chi_{\lambda} \uparrow$ when $d(\lambda)$ is even and $\tilde{\chi}_{\lambda} \uparrow=\left(\chi_{\lambda}+\chi_{\lambda}^{\prime}\right) \uparrow=\chi_{\lambda} \uparrow+\chi_{\lambda}^{\prime} \uparrow$ when $d(\lambda)$ is odd. If $\chi_{\lambda}$ is irreducible, then $\chi_{\lambda} \uparrow$ is irreducible by Proposition 3.3. Hence by Mackey's decomposition theorem and Frobenius reciprocity we obtain (cf. [3]):

$$
\left\langle\chi_{\lambda} \uparrow, \chi_{\lambda} \uparrow\right\rangle_{\widetilde{\Gamma}_{n}}=\left\langle\chi_{\lambda}, \chi_{\lambda}\right\rangle_{\widetilde{\Gamma}_{\lambda}}
$$

3.5. Spin character values. Let $\lambda=\left(\lambda_{\gamma_{0}}, \cdots, \lambda_{\gamma_{r}}\right)$ be a strict partition-valued function on $\Gamma^{*}$. Let $\rho=\rho^{0} \cup \cdots \cup \rho^{r}$ be the partition-valued function defined in Remark 3.4 such that $\left|\rho^{j}\right|=\left|\lambda_{\gamma_{j}}\right|$ for $j=0, \cdots, r$. From Schur's results in Theorem 3.1, one sees that if $\Delta_{\lambda_{\gamma_{j}}}\left(t_{\rho^{j}}\right)$ has a nonzero value, then $\rho^{j}$ must be in $\left[\lambda_{\gamma_{j}}\right]$ for $\gamma_{j} \in J_{\lambda}$, and $\rho^{j}$ must lie in $\mathcal{O S P}_{\left|\lambda_{\gamma_{j}}\right|}\left(\Gamma_{*}\right):=\mathcal{O} \mathcal{P}_{\left|\lambda_{\gamma_{j}}\right|}\left(\Gamma_{*}\right) \cap \mathcal{S} \mathcal{P}_{\left|\lambda_{\gamma_{j}}\right|}\left(\Gamma_{*}\right)$ for $\gamma_{j} \in J_{\lambda}^{\prime}$. Then we have the following result. 
Proposition 3.5. Let $\lambda=\left(\lambda_{\gamma}\right)_{\gamma \in \Gamma^{*}} \in \mathcal{S P}_{n}^{1}\left(\Gamma^{*}\right)$. If $\rho=\bigcup_{j=0}^{r} \rho_{\gamma_{j}} \in \mathcal{S P}_{n}^{1}\left(\Gamma_{*}\right)$ such that $\rho_{\gamma_{j}}$ lies in $\left[\lambda_{\gamma_{j}}\right]$ for $\gamma_{j} \in J_{\lambda}$, and $\rho_{\gamma_{j}}$ is in $\mathcal{O} \mathcal{S P} \mathcal{P}_{\left|\lambda_{\gamma_{j}}\right|}\left(\Gamma_{*}\right)$ for $\gamma_{j} \in J_{\lambda}^{\prime}$, then

$$
\prod_{\gamma \in J_{\lambda}^{\prime}}\left(\sum_{\rho_{\gamma} \in \mathcal{O} \mathcal{S P}_{\left|\rho_{\gamma}\right|}\left(\Gamma_{*}\right)} \frac{1}{Z_{\rho_{\gamma}}}\left|\prod_{c \in \Gamma_{*}} \gamma(c)^{l\left(\rho_{\gamma}(c)\right)} \Delta_{\lambda_{\gamma}}\left(t_{\rho_{\gamma}}\right)\right|^{2}\right)=1 .
$$

Proof. Write $m=\left|J_{\lambda}\right|$. Then we have (note: here $m$ is odd)

$$
\begin{aligned}
& \left\langle\chi_{\lambda} \uparrow, \chi_{\lambda} \uparrow\right\rangle_{\widetilde{\Gamma}_{n}-\Gamma \chi \widetilde{A}_{n}}
\end{aligned}
$$

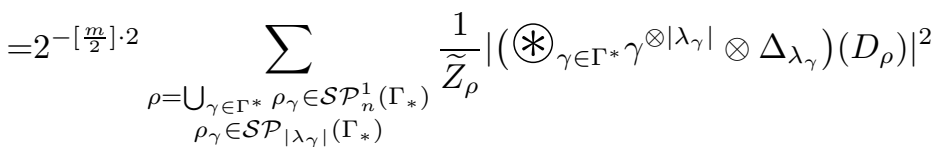

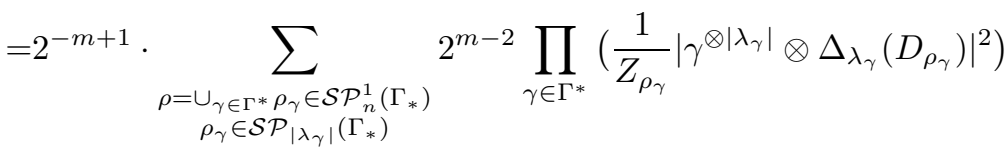

$$
\begin{aligned}
& =\frac{1}{2} \prod_{\gamma \in \Gamma^{*}}\left(\sum_{\bar{d}\left(\rho_{\gamma}\right)=\bar{d}\left(\lambda_{\gamma}\right), \rho_{\gamma} \in \mathcal{S} \mathcal{P}_{\left|\lambda_{\gamma}\right|}\left(\Gamma_{*}\right)} \frac{1}{Z_{\rho_{\gamma}}}\left|\gamma^{\otimes\left|\lambda_{\gamma}\right|} \otimes \Delta_{\lambda_{\gamma}}\left(D_{\rho_{\gamma}}\right)\right|^{2}\right) .
\end{aligned}
$$

As $Z_{\rho}=\prod_{\gamma \in \Gamma^{*}} Z_{\rho_{\gamma}}$ and $\widetilde{Z}_{\rho_{\gamma}}=2 Z_{\rho_{\gamma}}$ for $\gamma \in J_{\lambda}$, so (3.6) becomes

$$
\begin{aligned}
= & \frac{1}{2} \prod_{\gamma \in J_{\lambda}}\left(\sum_{\rho_{\gamma} \in \mathcal{S} \mathcal{P}_{\left|\lambda_{\gamma}\right|}^{1}\left(\Gamma_{*}\right)} \frac{2}{\widetilde{Z}_{\rho_{\gamma}}}\left|\gamma^{\otimes\left|\lambda_{\gamma}\right|} \otimes \Delta_{\lambda_{\gamma}}\left(D_{\rho_{\gamma}}\right)\right|^{2}\right) \\
& \cdot \prod_{\gamma \in J_{\lambda}^{\prime}}\left(\sum_{\rho_{\gamma} \in \mathcal{S} \mathcal{P}_{\left|\lambda_{\gamma}\right|}^{0}\left(\Gamma_{*}\right)} \frac{1}{Z_{\rho_{\gamma}}}\left|\gamma^{\otimes\left|\lambda_{\gamma}\right|} \otimes \Delta_{\lambda_{\gamma}}\left(D_{\rho_{\gamma}}\right)\right|^{2}\right) \\
= & 2^{m-1} \prod_{\gamma \in J_{\lambda}}\left\langle\gamma^{\otimes\left|\lambda_{\gamma}\right|} \otimes \Delta_{\lambda_{\gamma}}, \gamma^{\otimes\left|\lambda_{\gamma}\right|} \otimes \Delta_{\lambda_{\gamma}}\right\rangle_{\mathcal{S} \mathcal{P}_{\left|\lambda_{\gamma}\right|}^{1}\left(\Gamma_{*}\right)} \\
& \cdot \prod_{\gamma \in J_{\lambda}^{\prime}}\left(\sum_{\rho_{\gamma} \in \mathcal{O} \mathcal{S P}_{\left|\lambda_{\gamma}\right|}\left(\Gamma_{*}\right)} \frac{1}{Z_{\rho_{\gamma}}}\left|\gamma^{\otimes\left|\lambda_{\gamma}\right|} \otimes \Delta_{\lambda_{\gamma}}\left(D_{\rho_{\gamma}}\right)\right|^{2}\right) \\
= & \frac{1}{2} \prod_{\gamma \in J_{\lambda}^{\prime}}\left(\sum_{\rho_{\gamma} \in \mathcal{O} \mathcal{S P}_{\left|\lambda_{\gamma}\right|}\left(\Gamma_{*}\right)} \frac{1}{Z_{\rho_{\gamma}}}\left|\gamma^{\otimes\left|\lambda_{\gamma}\right|} \otimes \Delta_{\lambda_{\gamma}}\left(D_{\rho_{\gamma}}\right)\right|^{2}\right) .
\end{aligned}
$$

Thus it follows from [1] and Lemma 3.2 that

$$
\left\langle\chi_{\lambda} \uparrow, \chi_{\lambda} \uparrow\right\rangle_{\mathcal{O P}}\left(\Gamma_{*}\right)=\left\langle\chi_{\lambda} \uparrow, \chi_{\lambda} \uparrow\right\rangle_{\mathcal{S P}_{n}^{1}\left(\Gamma_{*}\right)}=\frac{1}{2} .
$$

Equations (3.6) and (3.7) imply that

$$
\prod_{\gamma \in J_{\lambda}^{\prime}}\left(\sum_{\rho_{\gamma} \in \mathcal{O} \mathcal{S} \mathcal{P}_{\left|\rho_{\gamma}\right|}\left(\Gamma_{*}\right)} \frac{1}{Z_{\rho_{\gamma}}}\left|\prod_{c \in \Gamma_{*}} \gamma(c)^{l\left(\rho_{\gamma}(c)\right)} \Delta_{\lambda_{\gamma}}\left(z^{p} t_{\rho_{\gamma}}\right)\right|^{2}\right)=1 .
$$



$\widetilde{\Gamma}_{n}$.

The following theorem gives the remaining part of the spin character table for

Theorem 3.6. Let $\lambda=\left(\lambda_{\gamma}\right)_{\gamma \in \Gamma^{*}} \in \mathcal{S P}_{n}^{1}\left(\Gamma^{*}\right)$ and $\rho \in \mathcal{S P}_{n}^{1}\left(\Gamma_{*}\right)$. (i) If $\rho=$ $\bigcup_{\gamma \in \Gamma^{*}} \rho_{\gamma}$ such that $\rho_{\gamma} \in\left[\lambda_{\gamma}\right]$ for $\gamma \in J_{\lambda}$ and $\rho_{\gamma} \in \mathcal{O S P}_{\left|\rho_{\gamma}\right|}\left(\Gamma_{*}\right)$ for $\gamma \in J_{\lambda}^{\prime}$, then

$$
\begin{aligned}
\chi_{\lambda} \uparrow\left(D_{\rho}^{ \pm}\right)= & \pm \prod_{\gamma \in \Gamma^{*}} \prod_{c \in \Gamma_{*}} \gamma(c)^{l\left(\rho_{\gamma}(c)\right)} \cdot \prod_{\gamma \in J_{\lambda}^{\prime}} \Delta_{\lambda_{\gamma}}\left(t_{\rho_{\gamma}}\right) \\
& \cdot(\sqrt{-1})^{\Sigma_{\gamma \in J_{\lambda}} \frac{\left|\lambda_{\gamma}\right|-l\left(\lambda_{\gamma}\right)+1}{2}} \sqrt{\frac{\prod_{\gamma \in J_{\lambda}} z_{\lambda_{\gamma}}}{2}},
\end{aligned}
$$

where the value of $\prod_{\gamma \in J_{\lambda}^{\prime}} \Delta_{\lambda_{\gamma}}\left(t_{\rho_{\gamma}}\right)$ is given by Schur Q-functions (see [1]).

(ii) $\chi_{\lambda} \uparrow\left(D_{\rho}^{ \pm}\right)=0$, otherwise.

Proof. (i) Let $T$ be a left coset of $\widetilde{\Gamma}_{\lambda}$ in $\widetilde{\Gamma}_{n}$ and let $K_{\rho}$ be the number of left cosets $T$ of $\widetilde{\Gamma}_{\lambda}$ in $\widetilde{\Gamma}_{n}$ such that $\left(g, t_{\rho}\right) T=T$. Then $t^{-1}\left(g, t_{\rho}\right) t \in \widetilde{\Gamma}_{\lambda}$ for any left coset representative $t \in T$. It follows from the formula of induced character that

$$
\begin{aligned}
\chi_{\lambda} \uparrow\left(D_{\rho}^{ \pm}\right) & = \pm \frac{1}{\left|\widetilde{\Gamma}_{\lambda}\right|} \sum_{T \in \widetilde{\Gamma}_{n} / \widetilde{\Gamma}_{\lambda}}\left(\sum_{t \in T} \circledast_{\gamma \in \Gamma^{*}}\left(\gamma^{\otimes\left|\lambda_{\gamma}\right|} \otimes \Delta_{\lambda_{\gamma}}\right)\left(t^{-1}\left(g, t_{\rho}\right) t\right)\right) \\
& = \pm K_{\rho} \cdot \circledast_{\gamma \in \Gamma^{*}}\left(\gamma^{\otimes\left|\lambda_{\gamma}\right|} \otimes \Delta_{\lambda_{\gamma}}\right)\left(g, t_{\rho}\right) \\
& = \pm K_{\rho} \cdot 2^{\frac{\left|J_{\lambda}\right|-1}{2}} \prod_{\gamma \in \Gamma^{*}}\left(\gamma^{\otimes\left|\lambda_{\gamma}\right|} \otimes \Delta_{\lambda_{\gamma}}\right)\left(D_{\rho_{\gamma}}^{+}\right) .
\end{aligned}
$$

In the above we have used $\chi_{\lambda} \uparrow\left(D_{\rho}^{-}\right)=-\chi_{\lambda} \uparrow\left(D_{\rho}^{+}\right)$, and the second line vanishes if $t_{\rho} \notin \widetilde{S}_{\lambda}$. Moreover, by [1],

$$
\gamma^{\otimes\left|\lambda_{\gamma}\right|} \otimes \Delta_{\lambda_{\gamma}}\left(D_{\rho_{\gamma}}^{+}\right)=\prod_{c \in \Gamma_{*}} \gamma(c)^{l\left(\rho_{\gamma}(c)\right)} \cdot \Delta_{\lambda_{\gamma}}\left(t_{\rho_{\gamma}}\right)
$$

So (3.9) is equal to

$$
\begin{aligned}
& = \pm K_{\rho} \cdot 2^{\frac{\left|J_{\lambda}\right|-1}{2}} \prod_{\gamma \in \Gamma^{*}} \prod_{c \in \Gamma_{*}} \gamma(c)^{l\left(\rho_{\gamma}(c)\right)} \cdot \prod_{\gamma \in \Gamma^{*}} \Delta_{\lambda_{\gamma}}\left(t_{\rho_{\gamma}}\right) \\
& = \pm K_{\rho} \cdot 2^{\frac{\left|J_{\lambda}\right|-1}{2}} \prod_{\gamma \in \Gamma^{*}} \prod_{c \in \Gamma_{*}} \gamma(c)^{l\left(\rho_{\gamma}(c)\right)} \cdot \prod_{\gamma \in J_{\lambda}^{\prime}} \Delta_{\lambda_{\gamma}}\left(t_{\rho_{\gamma}}\right) \\
& \cdot \prod_{\gamma \in J_{\lambda}}(\sqrt{-1})^{\frac{\left|\rho_{\gamma}\right|-l\left(\rho_{\gamma}\right)+1}{2}} \sqrt{\frac{\prod_{c \in \Gamma_{*}} z_{\rho_{\gamma}(c)}}{2}} \\
& = \pm K_{\rho} \prod_{\gamma \in \Gamma^{*}} \prod_{c \in \Gamma_{*}} \gamma(c)^{l\left(\rho_{\gamma}(c)\right)} \cdot \prod_{\gamma \in J_{\lambda}^{\prime}} \Delta_{\lambda_{\gamma}}\left(t_{\rho_{\gamma}}\right) \\
& \cdot(\sqrt{-1})^{\Sigma_{\gamma \in J_{\lambda}} \frac{|\lambda \gamma|-l(\lambda \gamma)+1}{2}} \sqrt{\frac{\prod_{\gamma \in J_{\lambda}} z_{\lambda_{\gamma}}}{2}} .
\end{aligned}
$$

(ii) Note that we can assume that $\rho=\bigcup_{\gamma \in \Gamma^{*}} \rho_{\gamma}$, where $\rho_{\gamma} \in \mathcal{S P} \mathcal{P}_{\left|\lambda_{\gamma}\right|}\left(\Gamma_{*}\right)$. Otherwise it is easy to see that $\chi_{\lambda} \uparrow(\rho)=0$. 
(1) If $\rho_{\gamma} \in \mathcal{O S P}_{\left|\rho_{\gamma}\right|}\left(\Gamma_{*}\right)$ for $\gamma \in J_{\lambda}^{\prime}$, we show that $\rho_{\gamma} \in\left[\lambda_{\gamma}\right]$ for $\gamma \in J_{\lambda}$. We denote $m=\left|J_{\lambda}\right|$. It follows from Proposition 3.5 that

$$
\begin{aligned}
& \left\langle\chi_{\lambda} \uparrow, \chi_{\lambda} \uparrow\right\rangle_{\mathcal{S} \mathcal{P}_{n}^{1}\left(\Gamma_{*}\right)} \\
= & 2^{m-1} \prod_{\gamma \in J_{\lambda}}\left(\sum_{\rho_{\gamma} \in \mathcal{S} \mathcal{P}_{\left|\lambda_{\gamma}\right|}^{1}\left(\Gamma_{*}\right)} \frac{1}{\widetilde{Z}_{\rho_{\gamma}}}\left|\prod_{c \in \Gamma_{*}} \gamma(c)^{l\left(\rho_{\gamma}(c)\right)} \Delta_{\lambda_{\gamma}}\left(z^{p} t_{\rho_{\gamma}}\right)\right|^{2}\right) \\
\geq & 2^{m-1} \prod_{\gamma \in J_{\lambda}}\left(\sum_{\rho_{\gamma} \in\left[\lambda_{\gamma}\right]} \frac{1}{Z_{\rho_{\gamma}}}\left|\prod_{c \in \Gamma_{*}} \gamma(c)^{l\left(\rho_{\gamma}(c)\right)} \Delta_{\lambda_{\gamma}}\left(t_{\rho_{\gamma}}\right)\right|^{2}\right) \\
= & 2^{m-1} \prod_{\gamma \in J_{\lambda}}\left(\sum_{\rho_{\gamma} \in\left[\lambda_{\gamma}\right]}\left(\prod_{c \in \Gamma_{*}} \frac{|\gamma(c)|^{2 l\left(\rho_{\gamma}(c)\right)}}{\left.z_{\rho_{\gamma}(c)} \zeta_{c}^{l\left(\rho_{\gamma}(c)\right)}\right)}\left|\sqrt{\frac{\prod_{c \in \Gamma_{*}} z_{\rho_{\gamma}(c)}}{2}}\right|^{2}\right)\right. \\
= & 2^{m-1} \prod_{\gamma \in J_{\lambda}}\left(\sum_{\rho_{\gamma} \in\left[\lambda_{\gamma}\right]} \frac{1}{2} \frac{\prod_{c \in \Gamma_{*}}|\gamma(c)|^{2 l\left(\rho_{\gamma}(c)\right)}}{\prod_{c \in \Gamma_{*}} \zeta_{c}^{l\left(\rho_{\gamma}(c)\right)}}\right) .
\end{aligned}
$$

Since $\rho_{\gamma} \in\left[\lambda_{\gamma}\right]$, then $l\left(\rho_{\gamma}\right)=l\left(\lambda_{\gamma}\right)$ and

$$
\gamma(c)^{l\left(\rho_{\gamma}(c)\right)}=\gamma^{\otimes l\left(\rho_{\gamma}(c)\right)} \cdot(\underbrace{c, \cdots, c}_{l\left(\rho_{\gamma}(c)\right)}) .
$$

Hence, we have that

$$
\begin{aligned}
& \prod_{c \in \Gamma_{*}} \gamma(c)^{l\left(\rho_{\gamma}(c)\right)=\gamma \otimes l\left(\rho_{\gamma}\right)} \cdot(\underbrace{c^{0}, \cdots, c^{0}}_{l\left(\rho_{\gamma}\left(c^{0}\right)\right)}, \cdots, \underbrace{c^{r}, \cdots, c^{r}}_{l\left(\rho_{\gamma}\left(c^{r}\right)\right)}) \\
= & \gamma^{\otimes l\left(\rho_{\gamma}\left(c^{0}\right)\right)} \otimes \cdots \otimes \gamma^{\otimes l\left(\rho_{\gamma}\left(c^{r}\right)\right)} \cdot(\underbrace{c^{0}, \cdots, c^{0}}_{l\left(\rho_{\gamma}\left(c^{0}\right)\right)}, \cdots, \underbrace{c^{r}, \cdots, c^{r}}_{l\left(\rho_{\gamma}\left(c^{r}\right)\right)}) \\
= & \gamma^{\otimes l\left(\rho_{\gamma}\right)} \cdot\left(c^{i_{1}}, c^{i_{2}}, \cdots, c^{\left.i_{l\left(\rho_{\gamma}\right)}\right)}\right.
\end{aligned}
$$

which satisfies that the number of $c^{j}(j=0, \cdots, r)$ in $\left\{c^{i_{1}}, c^{i_{2}}, \cdots, c^{i_{l\left(\rho_{\gamma}\right)}}\right\}$ is equal to $l\left(\rho_{\gamma}\left(c^{j}\right)\right)$. Clearly, $C_{\rho_{\gamma}}=\left(c^{i_{1}}, c^{i_{2}}, \cdots, c^{\left.i_{l\left(\rho_{\gamma}\right)}\right)}\right.$ is a conjugacy class of $\Gamma^{l\left(\lambda_{\gamma}\right)}$. Let $\zeta_{C_{\rho_{\gamma}}}$ be the order of the centralizer of an element in the conjugacy class $C_{\rho_{\gamma}}$ of $\Gamma^{l\left(\lambda_{\gamma}\right)}$; then $\zeta_{C_{\rho_{\gamma}}}=\prod_{c \in \Gamma_{*}} \zeta_{c}^{l\left(\rho_{\gamma}(c)\right)}$. As $\rho_{\gamma} \in\left[\lambda_{\gamma}\right]$, so $C_{\rho_{\gamma}}$ can run through all conjugacy classes in $\Gamma^{l\left(\lambda_{\gamma}\right)}$. For example, if we assume $\left[\lambda_{\gamma}\right]=\left\{\rho_{\gamma} \in \mathcal{S} \mathcal{P}_{11}^{1}\left(\Gamma_{*}\right) \mid \lambda_{\gamma}=(5,4,2)\right\}$, then $\rho_{\gamma}=\left(5_{\square_{1}}, 4_{\square_{2}}, 2_{\square_{3}}\right)$ and each $\square_{j}$ can run from $c^{0}$ to $c^{r}$. So $C_{\rho_{\gamma}}=\left(\square_{1}, \square_{2}, \square_{3}\right)$ 
runs through all conjugacy classes of $\Gamma^{3}$. Subsequently 3.11) becomes

$$
\begin{aligned}
& =2^{m-1} \prod_{\gamma \in J_{\lambda}}\left(\sum_{C_{\rho_{\gamma}} \in\left(\Gamma^{l\left(\lambda_{\gamma}\right)}\right)_{*}} \frac{1}{2 \zeta_{\rho_{\rho_{\gamma}}}}\right. \\
& \left.\cdot\left|\gamma^{\otimes l\left(\rho_{\gamma}\left(c^{0}\right)\right)} \otimes \cdots \otimes \gamma^{\otimes l\left(\rho_{\gamma}\left(c^{r}\right)\right)} \cdot(\underbrace{c^{0}, \cdots, c^{0}}_{l\left(\rho_{\gamma}\left(c^{0}\right)\right)}, \cdots, \underbrace{c^{r}, \cdots, c^{r}}_{l\left(\rho_{\gamma}\left(c^{r}\right)\right)})\right|^{2}\right) \\
& =2^{m-1} \prod_{\gamma \in J_{\lambda}}\left(\sum_{C_{\rho_{\gamma}} \in\left(\Gamma^{l\left(\lambda_{\gamma}\right)}\right)_{*}} \frac{1}{2 \zeta_{C_{\rho_{\gamma}}}} \cdot \mid \gamma^{\otimes l\left(\rho_{\gamma}\right)}\left(c^{i_{1}}, c^{i_{2}}, \cdots,\left.c^{\left.i_{l\left(\rho_{\gamma}\right)}\right)}\right|^{2}\right)\right. \\
& =2^{m-1} \prod_{\gamma \in J_{\lambda}}\left(\frac{1}{2} \sum_{C_{\rho_{\gamma}} \in\left(\Gamma^{l\left(\lambda_{\gamma}\right)}\right)_{*}} \frac{1}{\zeta_{C_{\rho_{\gamma}}}}\left|\gamma^{\otimes l\left(\lambda_{\gamma}\right)}\left(C_{\rho_{\gamma}}\right)\right|^{2}\right) \\
& =2^{m-1} \prod_{\gamma \in J_{\lambda}} \frac{1}{2}\left\langle\gamma^{\otimes l\left(\lambda_{\gamma}\right)}, \gamma^{\otimes l\left(\lambda_{\gamma}\right)}\right\rangle_{\Gamma^{l\left(\lambda_{\gamma}\right)}}=\frac{1}{2},
\end{aligned}
$$

which forces $\chi_{\lambda} \uparrow\left(D_{\rho}^{ \pm}\right)=0$ if $\rho_{\gamma} \notin\left[\lambda_{\gamma}\right]$ for $\gamma \in J_{\lambda}$.

(2) If $\rho_{\gamma} \notin \mathcal{O S P} \mathcal{P}_{\left|\lambda_{\gamma}\right|}\left(\Gamma_{*}\right)$ for $\gamma \in J_{\lambda}^{\prime}$, then there is at least one $\rho_{\gamma}$ not in $\mathcal{O P} \mathcal{P}_{\left|\lambda_{\gamma}\right|}\left(\Gamma_{*}\right)$ for $\gamma \in J_{\lambda}^{\prime}$. Meanwhile, $\Delta_{\lambda_{\gamma}}$ is a double spin character when $\gamma \in J_{\lambda}^{\prime}$, so we have $\Delta_{\lambda_{\gamma}}\left(t_{\rho_{\gamma}}\right)=0$, thus $\chi_{\lambda} \uparrow\left(D_{\rho}^{ \pm}\right)=0$.

If we plug $\chi_{\lambda} \uparrow$ into $\left\langle\chi_{\lambda} \uparrow, \chi_{\lambda} \uparrow\right\rangle=1 / 2$, we then get $K_{\rho}=1$ in (3.9).

This completes the proof.

\section{ACKNOWLEDGMENTS}

The second author gratefully acknowledges the partial support of Max-Planck Institut für Mathematik in Bonn, Simons Foundation, NSFC and NSF during this work.

\section{REFERENCES}

[1] Igor B. Frenkel, Naihuan Jing, and Weiqiang Wang, Twisted vertex representations via spin groups and the McKay correspondence, Duke Math. J. 111 (2002), no. 1, 51-96, DOI 10.1215/S0012-7094-02-11112-0. MR1876441 (2003d:17036)

[2] Peter N. Hoffman and John F. Humphreys, Hopf algebras and projective representations of $G\left\{S_{n}\right.$ and $G \nmid A_{n}$, Canad. J. Math. 38 (1986), no. 6, 1380-1458, DOI 10.4153/CJM-1986-070-1. MR.873418(88h:20014)

[3] X. Hu and N. Jing, Spin characters of generalized symmetric groups, Monatsh. Math. 173 (2014), 495-518. doi:10.1007/s00605-013-0525-y.

[4] Nai Huan Jing, Vertex operators, symmetric functions, and the spin group $\Gamma_{n}$, J. Algebra 138 (1991), no. 2, 340-398, DOI 10.1016/0021-8693(91)90177-A. MR1102815 (92e:17033)

[5] Tadeusz Józefiak, A class of projective representations of hyperoctahedral groups and Schur Q-functions, Topics in algebra, Part 2 (Warsaw, 1988), Banach Center Publ., vol. 26, PWN, Warsaw, 1990, pp. 317-326. MR1171281 (93e:20016)

[6] I. G. Macdonald, Symmetric functions and Hall polynomials, 2nd ed., Oxford Mathematical Monographs, The Clarendon Press Oxford University Press, New York, 1995. With contributions by A. Zelevinsky; Oxford Science Publications. MR.1354144 (96h:05207)

[7] A. O. Morris, The spin representation of the symmetric group, Proc. London Math. Soc. (3) 12 (1962), 55-76. MR.0136668 (25 \#133)

[8] Alun O. Morris and Huw I. Jones, Projective representations of generalized symmetric groups, Sém. Lothar. Combin. 50 (2003/04), Art. B50b, 27 pp. (electronic). MR2049553 (2005c:20022) 
[9] Maxim Nazarov, Young's symmetrizers for projective representations of the symmetric group, Adv. Math. 127 (1997), no. 2, 190-257, DOI 10.1006/aima.1997.1621. MR.1448714 $(98 \mathrm{~m}: 20019)$

[10] E. W. Read, The $\alpha$-regular classes of the generalized symmetric group, Glasgow Math. J. 17 (1976), no. 2, 144-150. MR.0412267 (54 \#393)

[11] I. Schur, Über die Darstellung der symmertrischen und der alternierenden Gruppe durch gebrochene lineare Substitutionen, J. Reine Angew. Math. 139 (1911), 155-250.

[12] Jean-Pierre Serre, Représentations linéaires des groupes finis (French), Third revised edition, Hermann, Paris, 1978. MR543841 (80f:20001)

[13] W. Specht, Eine Verallgemeinerung der symmetrischen Gruppen, Schriffen Math. Sem. Berlin 1 (1932), 1-32.

[14] David B. Wales, Some projective representations of $S_{n}$, J. Algebra 61 (1979), no. 1, 37-57, DOI 10.1016/0021-8693(79)90304-1. MR554850 (81f:20015)

School of Mathematics and Computer Science, Jianghan University, Wuhan 430056 , People's Republic of China

E-mail address: xiaolihumath@163.com

Department of Mathematics, North Carolina State University, Raleigh, North CarOLINA 27695

E-mail address: jing@math.ncsu.edu 